\title{
ON THE LINK BETWEEN NORTHERN FENNOSCANDIAN CLIMATE AND LENGTH OF THE QUASI-ELEVEN-YEAR CYCLE IN GALACTIC COSMIC-RAY FLUX
}

\author{
M. G. OGURTSOV ${ }^{1}$, H. JUNGNER ${ }^{2}$, G. E. KOCHAROV ${ }^{1}$, M. LINDHOLM ${ }^{3}$, \\ M. ERONEN ${ }^{4}$ and YU. A. NAGOVITSYN 5 \\ ${ }^{1}$ A.F. Ioffe Physico-Technical Institute of Russian Academy of Sciences, 194021, \\ Polytechnicheskaya 26, St.-Petersburg, Russia \\ (e-mail: maxim.ogurtsov@pop.ioffe.rssi.ru) \\ ${ }^{2}$ University of Helsinki, Dating Laboratory, Gustav Hällströminkatu street 2, \\ POB 64, FIN-00014, Helsinki, Finland \\ ${ }^{3}$ Saima Center for Environmental Sciences, Linnankatu 11, FIN-00014, Savonlinna, Finland \\ ${ }^{4}$ University of Helsinki, Department of Geology, Gustav Hällströminkatu 2, \\ POB 64, FIN-00014, Helsinki, Finland \\ ${ }^{5}$ Central (Pulkovo) Astronomic Observatory, 196140, Pulkovskoe shosse 65/1, \\ St.-Petersburg, M-140, Russia
}

(Received 6 November 2002; accepted 2 September 2003)

\begin{abstract}
Bidecadal fluctuations in terrestrial climate were analyzed. It was shown that this variability might arise if Earth's climate reacts to galactic cosmic-ray intensity, integrated over its full quasi-11-year cycle. It was further shown that this integral effect should also lead to an effective link between climate and the duration of the quasi-11-year cycle in cosmic ray flux. That, in turn, must result in appearance of some connection between climate and the length of the solar cycle, which is currently a topic of active debate. Analyses of temperature proxies, obtained for northern Fennoscandia, confirmed the connection of the climate in this region and the length of the cycle in galactic cosmic-ray intensity. Decadal and bidecadal variability of integrated cosmic-ray flux was quantitatively estimated.
\end{abstract}

\section{Introduction}

Evidence linking solar activity and terrestrial climate has recently been presented by, e.g., Labitzke (1987), Reid (1991), Friis-Christensen and Lassen (1991), Pudovkin and Raspopov (1992), Veretenenko and Pudovkin (1995), Beer et al. (1994), Pudovkin and Veretenenko (1996), Svensmark and Friis-Christensen (1997), Pudovkin and Morozova (1998), Ogurtsov et al. (2002), and many others. These results show the reality of the link between the Sun's activity and climate, although many problems still remain and further studies are needed (see, e.g., Rind, 2002). In this work, we analyze two critical features of a possible solar-climatic relationship:

(1) The connection between long-term changes of terrestrial climate and solar cycle length (SCL). Friis-Christensen and Lassen (1991) linked SCL and global 
temperatures. Although the contribution of the effect caused by SCL variations in global climate change is currently actively debated (Damon and Peristykh, 1999; Laut and Gundermann, 2000), the existence of such a link has been confirmed for regional temperatures (Ulich, 1996; Alvarez Pomarez and Perez Doval, 2000; Zhou and Butler, 1998; Butler, 1994).

(2) Substantial bidecadal climate variability. Oscillations of periods close to 22 years were found in the rhythm of droughts in western USA (Mitchell, Stockton, and Meko, 1979), in temperatures of central England (Burroughs, 1994), in Greenland oxygen isotope records (Hibler and Johnsen, 1979), in dendrochronological series from northern Fennoscandia (NF) and Kola peninsula (Raspopov et al., 1998), in many Russian tree-ring records (Bitvinskas, 1971; Kocharov et al., 1986; Dergachev, Kocharov, and Stupneva, 1978), in Swiss temperature and humidity (Pudovkin and Morozova, 1999), and in experimentally measured global temperature (see Peristykh and Damon, 1998). It should be noted that the bidecadal variability in global climate is approximately of the same magnitude as the decadal one (Peristykh and Damon, 1998) while the 22-year cycle in sunspot activity is weak and its amplitude is no more than $10 \%$ of the present sunspot activity level (Mursula, Usoskin, and Kovaltsov, 2001). The connection between bidecadal climatic variation and the 22-year solar cycle (Hale cycle) seems very plausible, and in some cases it is convincingly confirmed by phase analysis (Mitchell, Stockton, and Meko, 1979). Estimations of Peristykh and Damon (1998) showed that in order to explain the observed amplitude of quasi-22-year periodicity in global temperatures, the irradiance change during the 22-year cycle should be quite strong, greater by a factor 1.5 compared to the 11 -year cycle. However, the 22-year cycle in sunspot activity is much weaker than the 11-year Schwabe cycle (Mursula, Usoskin, and Kovaltsov, 2001). This indicates that the Hale cycle in solar irradiance is likely rather weak, while the quasi-22-year periodicity in the terrestrial climate is at least of the same order of magnitude as the Schwabe cycle. Therefore, it is not easy to ascribe relatively strong bidecadal climatic variations to the oscillations of solar radiation. The 22-year cycle manifests itself in geomagnetic activity (Russell and Mulligan, 1995; Rivin, 1996). But this manifestation is weak, thus its analysis requires special filtration (see Rivin, 1996). We consider the galactic cosmic rays as the more probable cause for the quasi-22-year climatic variation, although the contribution of some other sources, such as 22-year variation of solar wind and interplanetary magnetic field (Baranyi, Ludmany, and Coffey, 1998) and precipitation of particles in auroral zone (Veretenenko and Pudovkin, 1997) cannot be excluded. The 22-year cycle is seen in the GCR intensity, since during solar cycles with negative polarity of the Sun's northern polar field (field is directed into the Sun) cosmic-ray time dependence has a peaked form and during cycles with positive polarity (field is directed out of the Sun) it has a plateau form. Such an effect is caused by the difference in cosmic ray drift directions during positive and negative phases of the magnetic cycle. The time behavior of galactic proton flux 


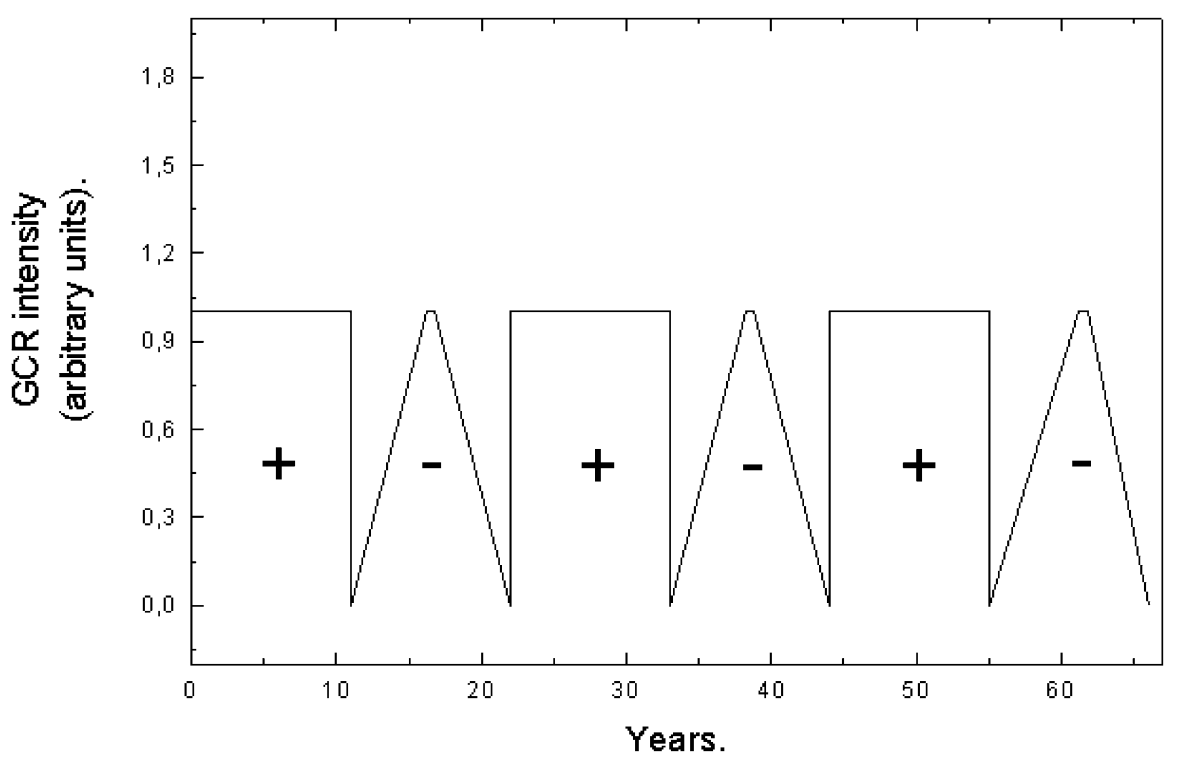

Figure 1. Idealized mathematical model of GCR intensity variations in 11-year and 22-year cycles after Peristykh and Damon (1998).

was theoretically investigated by Jokipii (1991), and its idealized form, taken from Peristykh and Damon (1998), is plotted in Figure 1.

It is evident that integrated cosmic-ray flux during the plateau phase of the cycle is twice as large as during the peaked phase. Clearly, if the Earth's atmosphere reacts effectively to the cosmic-ray flux integrated over the corresponding cycle, appreciable bidecadal variation in climate may arise. This variation should be more pronounced at high latitudes, because cosmic rays are more intense in the polar regions. Soviet dendrochronologists (Kocharov et al., 1986; Bitvinskas, 1971) have shown this latitudinal dependence. Analyzing rhythms in tree-ring time series along Murmansk-Carpathian profile (about 40 chronologies), Kocharov et al. (1986) presented evidence that bidecadal variability is stronger at higher latitudes and decadal variability at lower latitudes of the northern hemisphere. Figure 2 illustrates this result.

The tendency of the variation period to increase with increasing latitude is seen in Figure 2, although the scatter of points is appreciable. Naturally, the spatial distribution of the 22-year and the 11-year climatic cyclicity is more complicated, because atmospheric circulation, also affected by solar activity, can distort the latitudinal dependence. However, climatic records from many geographical locations agree with the tendency established by the above authors.

The Sun can affect the Earth's climate both directly, via total and ultraviolet radiation, SCR, solar wind and interplanery magnetic field, as well as indirectly, via modulation of GCR. The plausible mechanisms of solar impact on climate are the following: 


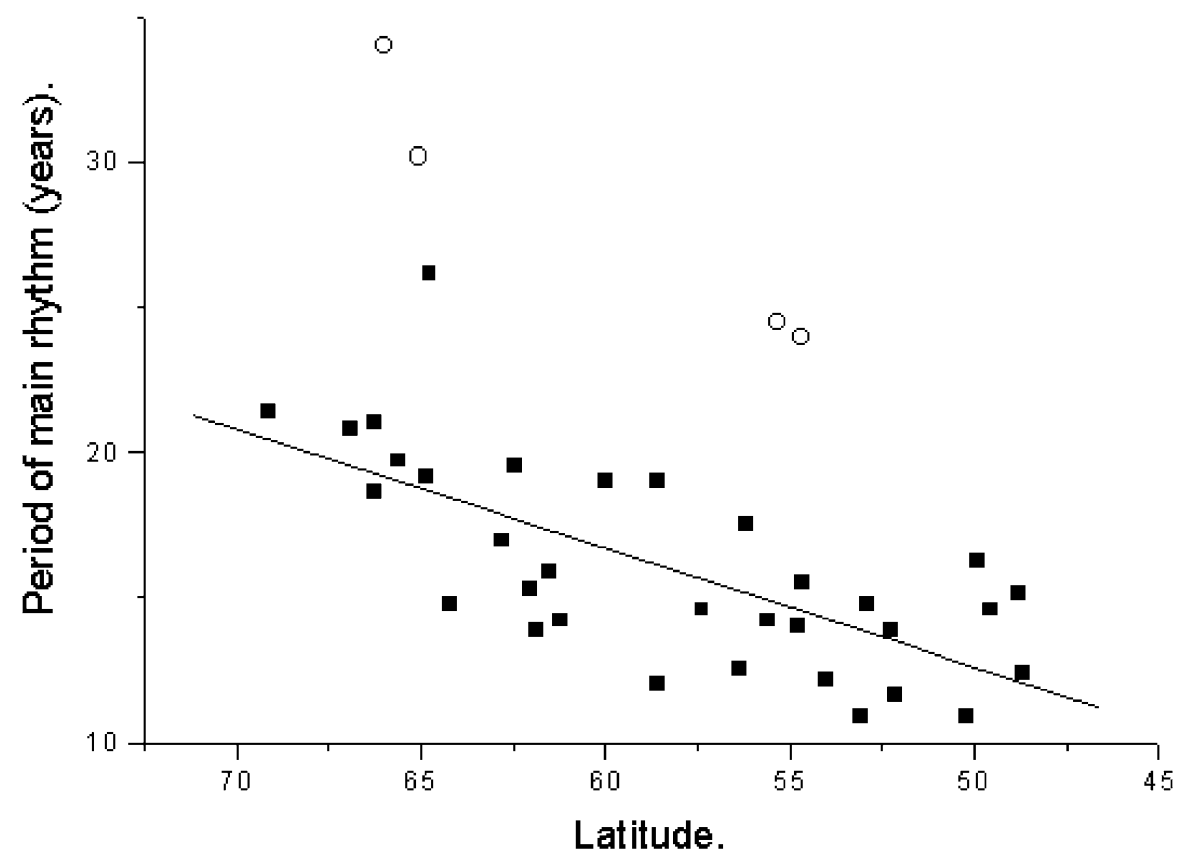

Figure 2. Latitudinal dependence of the period of rhythm of pine growth along the Murmansk-Carpathians profile after Kocharov et al. (1986). Squares - normal conditions, open circles - bog conditions.

(1) Dynamic mechanism of the Sun-climate connection takes into account the possible influence of variations of solar ultraviolet (UV) radiation on atmospheric dynamics and, hence, on climate. Models, linking changes in solar UV flux with the circulation of lower troposphere were suggested by Haigh (1996) and Shindell et al. (1999).

(2) Optical mechanism, which takes into account changes of atmospheric transparency caused by changes in fluxes of galactic cosmic rays (GCR) and solar cosmic rays (SCR), consisting mainly of energetic protons (energies up to few $\mathrm{GeV}$ ), can reach even the Earth's surface. Their fluxes change substantially with solar activity and can influence atmospheric opacity in two ways. The first is connected with the changes in atmospheric chemistry. The SCR and GCR particles react with $\mathrm{N}_{2}$ and $\mathrm{O}_{2}$, which lead to their dissociation and ionization. Ions of $\mathrm{N}_{2}^{+}$, $\mathrm{O}_{2}^{+}, \mathrm{N}^{+}, \mathrm{O}^{+}$are formed and they are involved in a complex of photochemical reactions, which produce nitrogen oxide, $\mathrm{NO}$. $\mathrm{NO}$ and atomic oxygen $\mathrm{O}$ effectively destroy ozone. Hence, the input of high-energy particles into the atmosphere causes destruction of ozone and the generation of $\mathrm{NO}_{2}$ (Pudovkin and Raspopov, 1992). Such changes are particularly strong during proton events. For example, on 4 August of 1972 , at $30-35 \mathrm{~km}$ altitude, the concentration of ozone decreased ten times and the concentration of $\mathrm{NO}_{2}$ increased by factor 2. Inasmuch as $\mathrm{NO}_{2}$ absorbs intensively solar radiation in the green and blue part of the spectrum, the 
irradiance at the Earth's surface decreases. Ultraviolet flux increases, due to ozone depletion of the stratosphere, and the radiation balance of the atmosphere changes, which may result in changes in atmospheric circulation. It should be noted that ozone depletion probably leads to the cooling of the Earth's surface, because the greenhouse effect of ozone exceeds the effect of UV heating (Larin, 2002). Thus, besides changes in the circulation pattern, variation in the chemical composition of the atmosphere, caused by input of energetic particles, can cool the lower troposphere. A change of the temperature altitude profile in the atmosphere, caused by high-energy particles, is described by Pudovkin and Dementeeva (1997).

(3) Electric mechanism is connected with GCR. Galactic cosmic rays, penetrating into the lower atmosphere, cause ionization in the troposphere, change electric potential between the Earth and the ionosphere, and influence the global electric chain. The global electric circuit can also be modulated by solar wind (Tinsley, 2000). These effects lead to variation in electric properties of cloud droplets. Changes of the electric state of clouds can cause respective change in cloud microphysics and, as a result, in substantial change in tropospheric dynamics (Tinsley and Deen, 1991; Tinsley, 2000; Carslaw, Harrison, and Kirkby, 2002). The connection between cosmic rays and cloudiness was proposed by Svensmark and Friis-Christensen (1997), who used data from geostationary satellites. A detailed discussion of the GCR-cloudiness relationship one can find in the work of Palle and Butler (2002). The role of storm clouds, more strongly connected with the electric state of the atmosphere, was emphasized by Ermakov (2000) and Stozhkov, Ermakov, and Pokrevski, (2001).

It may be summarized that the flux of energetic particles, which is strongly modulated by solar activity, can affect Earth's climate also via ionization in the atmosphere. Significant correlation between cloud cover and GCR intensity found by Svensmark and Friis-Christensen (1997) for global low cloudiness is the likely result of such reactions. The influence of cosmic rays on atmospheric ionization is now considered a more plausible mechanism to provide this link (Marsh and Svensmark, 2000). Observations show that the rate of condensation of aerosol by electrically charged water droplets is considerably higher than by neutral ones. A significant increase of aerosol concentration at latitudes of $15-25 \mathrm{~km}$ occurred after the solar proton event on 16 February 1984, as shown by Raspopov, Shumilov, and Kasatkina, (1998). Therefore, the change of cosmic-ray intensity causes respective changes in atmospheric ionization, in the rate of aerosol generation, and finally, in the rate of cloud formation. Both cloudiness and stratospheric aerosol concentration (termed by Raspopov, Shumilov, and Kasatkina (1998) as 'gray filter') can directly modulate background solar radiation, and, as a result, terrestrial climate. Apparent negative correlation between solar radiation input to the lower atmosphere and SCR and GCR intensity, found by Veretenenko and Pudovkin (2000), proves the existence of this relationship. Thus, the Sun can influence the Earth's climate both directly, via energetic protons of solar flares and particularly via ultraviolet radiation, and indirectly, via modulation of GCR intensity. 
In the present work, we continue the investigation of the Sun's modulation of climate, taking into account the features of a possible solar-climatic link as described above.

\section{Solar Effects on Climatic Proxies for Northern Fennoscandia}

It is important that a quasi-22-year variation is present in the earthly climate and that it is more distinct in the northern part of the hemisphere. Such a dependence can arise if the terrestrial climate system is sensitive to the GCR intensity integrated over individual quasi eleven-year cycles. In order to estimate the possible consequences of the SCL-climate relationship let us analyze climatic proxies obtained for northern Fennoscandia (NF), a reconstruction of July temperature in its continental part (Lindholm et al., 1999; Lindholm and Eronen, 2000) and reconstruction of mean growing season (April-September) temperature in its maritime part (Briffa et al. 1992). These series are plotted in Figures 3(a) and 3(b) (last three centuries). Lindholm and Eronen (2000) used ring widths of Scots pine (Pinus sylvestris L.) to reconstruct mid-summer (July) temperatures in northern Fennoscandia since 50 A.D. The chronology was constructed from 93 living trees and 275 dead trees (construction and subfossil timber) collected between $68^{\circ}$ and $70^{\circ} \mathrm{N}$, $20^{\circ}$ and $30^{\circ}$ E. The data and chronology building have been described by Eronen, Zetterberg, and Lindholm (1996) and Lindholm et al. (1996, 1999). Over one-half of the dependent climate variance was retrieved in the final reconstruction model.

Both of these series contain significant century scale (55-130 years) variability, which represents about $10 \%$ of the total variance. Fourier spectra of these data sets also show concentration of variance in 17-27 year range, an expected result from interference between decadal and bidecadal variations and the 30-33-year cycle of Bruckner. The centennial variations, wavelet filtered in the 54-147-year scale band (MHAT basis was used, see details of the method in Ogurtsov et al., 2001, 2002), are shown in Figures 3(c) and 3(d) together with the corresponding variation of Wolf numbers.

Figures 3(c) and 3(d) show that century-type cycles in temperature records and sunspot numbers correlate well, although this relationship shows an unphysical phase shift. It is seen that century-type climatic cycles outstrip the solar cycle by 15-20 years, the effect similar to the one found by Friis-Christensen and Lassen (1991) for a global hemispheric temperature. Friis-Christensen and Lassen solved the problem by using SCL as an indicator of the Sun's activity instead of sunspot numbers. It was shown by Solanki et al. (2002), Hathaway, Wilson, and Reichman, (2002) that the solar cycle length record precedes solar cycle amplitude by 1-3 cycles. Thus, a comparison was carried out between century-type variations of NF temperature and the length of quasi 11-year solar cycles, calculated between minima and maxima of solar activity. For the determination of the solar cycle duration we simply used dates of solar activity maxima and minima (ftp//ftp.ngdc.noaa.gov/ 


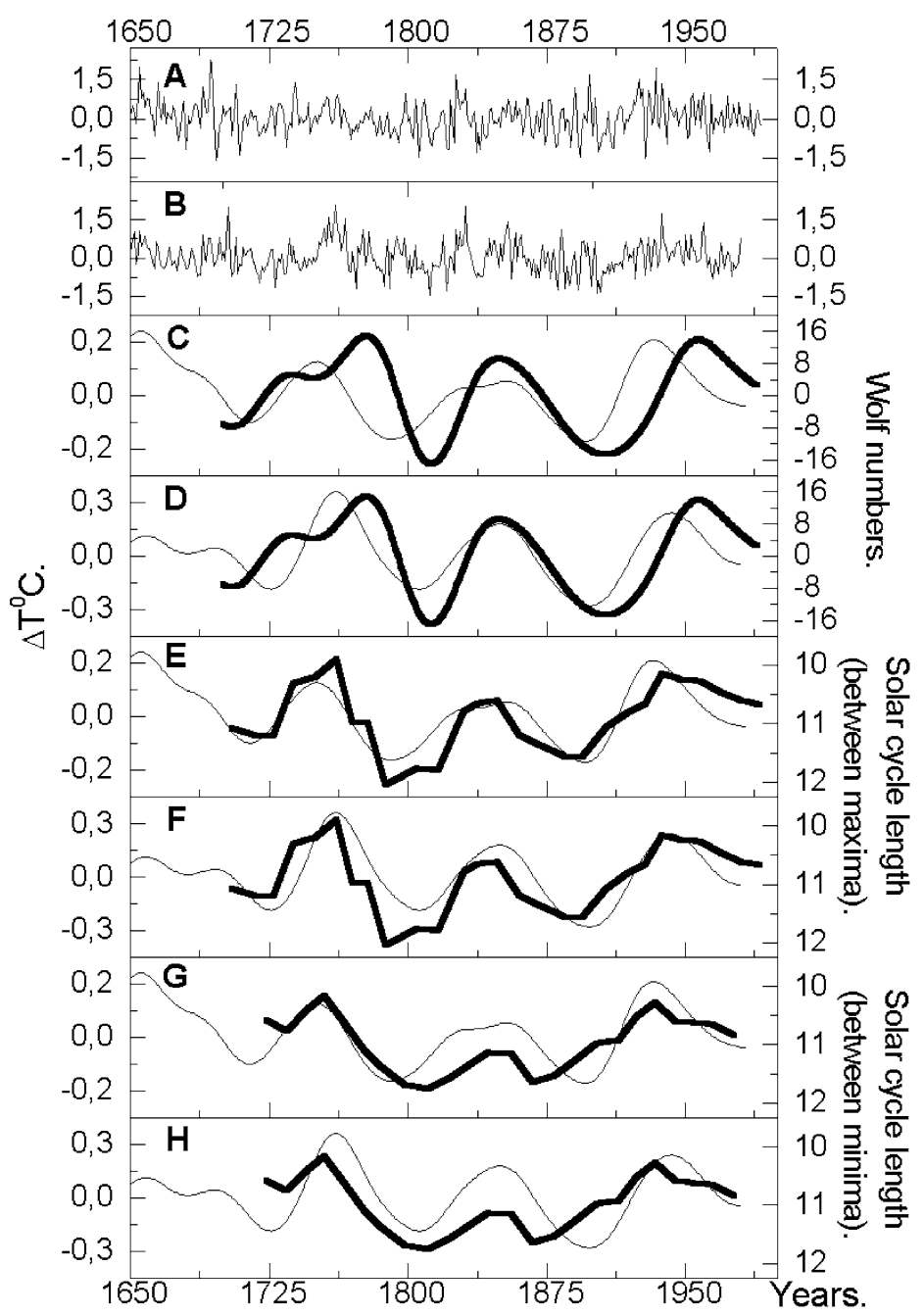

Figure 3. (A) Temperature in continental part of NF (raw data). (B) Temperature in maritime part of NF (raw data). (C) Wavelet filtered in 54-147-yr scale band (thin line) and smoothed SCL determined between maxima of solar activity (thick curve). (D) Temperature in maritime part of NF wavelet filtered in 54-147-yr scale band (thin line) and smoothed SCL determined between maxima of solar activity (thick curve). (E) Temperature in continental part of NF wavelet filtered in 54-147-yr scale band (thin line) and smoothed SCL determined between maxima of solar activity (thick curve). (F) Temperature in maritime part of NF wavelet filtered in 54-147-yr scale band (thin line) and smoothed SCL determined between minima of solar activity (thick curve). 
STP/SOLAR_DATA/SUNSPOT_NUMBERS/maxmin). Cycle lengths, determined in this way, were averaged over five points. Figure $3(\mathrm{e}-\mathrm{h})$ shows that the phase relationship between century-scale oscillation in NF climate and SCL is quite good. The SCL-temperature link is stronger, if the cycle duration is calculated between Wolf number maxima. Coefficients of correlation between the centennial cycle in continental and maritime parts of NF and SCL, determined between solar activity maxima, are 0.82 and 0.75 , while for SCL, determined between minima, they are 0.69 and 0.52 , respectively. Taking into account that significant correlation between smoothed SCL and tree-ring data from northern Finland has already been found by Zhou and Butler (1998), we can consider the SCL-climate link as reliably established for northern Fennoscandia. The cycles of GCR flux roughly correspond to sunspot cycles determined between maxima. Thus Figure 2 shows that the connection is stronger with the length of the cycle in GCR flux than with that in the sunspot cycle. This is considered as evidence that the duration of quasi elevenyear cycle in GCR intensity does affect NF climate. It should be noted that when we analyze the selected century-long periodicity (about $10 \%$ of full temperature variance) its link with the length of solar (GCR intensity) cycle appears rather naturally - in order to obtain quite apparent correlation it is enough to average SCL data by 5 points.

\section{Estimation of Integrated Effects of Galactic Cosmic Rays}

The existence of 22-year variation in many climatic series demonstrates the influence of charged particles (Vitinski, Ohl, and Sazonov, 1976), which may also provide a clue to the correlation between the solar cycle length and air temperature. Solanki, Schüssler, and Fligge (2000) and Solanki et al. (2002) showed that the secular variation of the solar open magnetic flux is affected by SCL while the solar open magnetic flux modulates the flux of GCR (Cane et al., 1999). Because the interplanetary magnetic field effectively modulates GCR intensity, galactic cosmic rays have been presented as a possible link between climate and SCL (Solanki, Schüssler, and Fligge, 2000). Raspopov et al. (1997) have noted the connection between the length of solar cycle and its intensity. It is known that shorter cycles tend to be more intensive, their maximal sunspot index reaching higher values (see Hoyng, 1993; Vitinski, Ohl, and Sazonov, 1976), although scatter is large Hathaway, Wilson and Reichman, (2002) found the correlation relatively weak (the correlation coefficient -0.27 for 21 cycles). Hence, the idea of Raspopov et al. (1997) draws attention to the link of solar activity distribution within a cycle with the cycle's duration.

Next we evaluate the connection between GCR intensity, integrated over an individual Schwabe cycle, and the length of this cycle. An idealized form of a quasi-11-year cycle in sunspot numbers is described by a formula suggested by Stewart and Panofski (1932): 


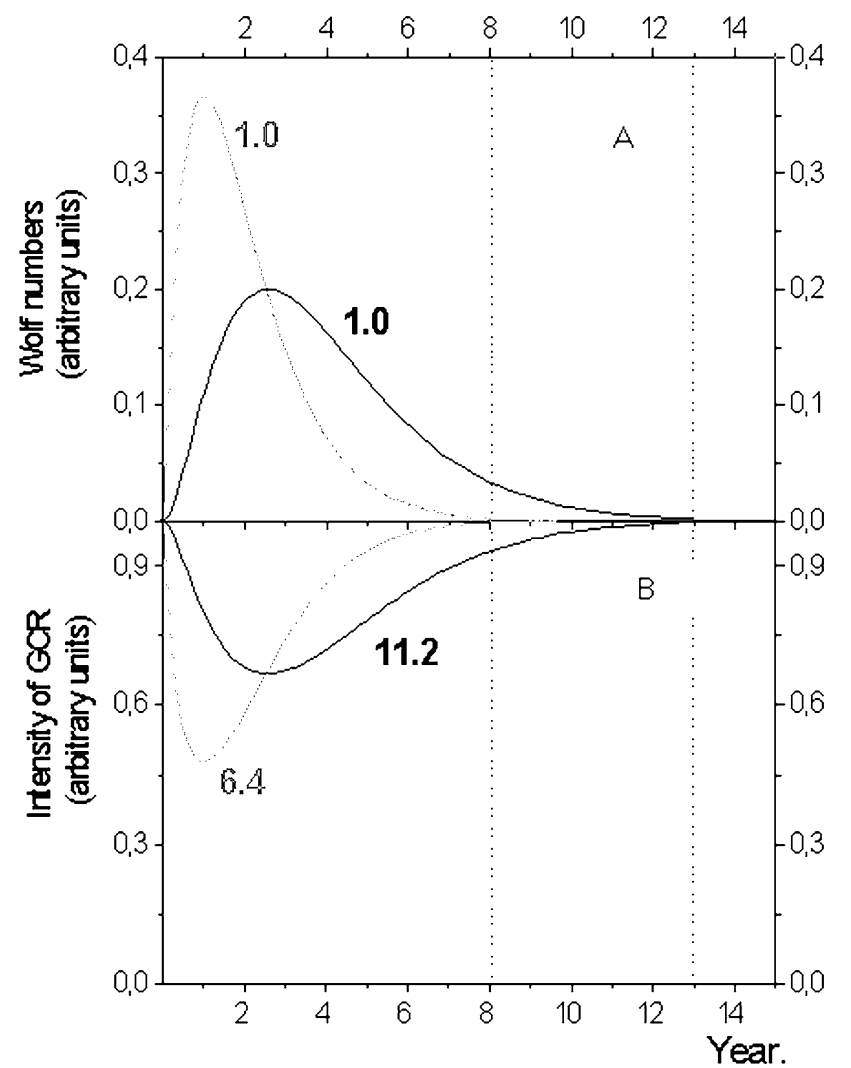

Figure 4. (A) Idealized mathematical model of quasi-11-year sunspot cycle (short cycle of 8 year length - dotted curve, long cycle of 13 year length - black curve), vertical dotted lines mark the length of cycle, which determines the range of integration. (B) Idealized mathematical model of quasi-11-year cycle in galactic cosmic-ray flux, modulated by solar cycles plotted in (A) (short cycle - dotted curve, long cycle - black curve).

$$
W(t)=W_{0} t^{a} \exp (-b t) .
$$

Two cycles, calculated by this formula are shown in Figure 4(A) - a shorter one (8 years length, $W_{0}=a=b=1.0$ ) and a longer one (13 years length, $W_{0}=0.22$, $a=1.8, b=0.7)$.

As was discussed above, the shorter one is more intensive - its maximum Wolf number is substantially larger than for the longer cycle. The time behavior of GCR intensity, modulated by solar activity, can be roughly estimated using a formula of Charakhchian et al. (1976):

$$
I(t)=I_{0} \exp \left(-A \eta(t)^{0.8} \phi(t)^{-1.2}\right) \approx I_{0} \exp \left(-A W(t)^{0.8}\right),
$$

where $I_{0}$ is the non-modulated flux of GCR, $\eta$ is the mean number of sunspot groups, $\phi$ their mean heliolatitude, and $W$ the Wolf number. 
The intensity of GCR, corresponding to two solar cycles (Figure 4(A)) and calculated by formula (2) is shown in Figure 4(B). The value of the Wolf number, integrated over the cycle, is equal for two cycles shown in Figure 4(A) (1.0 for both cycles). The other result we have for respective cycles in GCR intensity (Figure 4(B)). The integral over the shorter cycle is 6.4 while the integral over the longer cycle is more than 11.2 and thus the difference is evident. This means that even if amplitudes of shorter cycles are higher and the value, integrated over cycle, is close to be constant, the corresponding integral values of cycles in GCR flux may be appreciably different. Therefore, the value of GCR intensity, integrated over the full quasi 11-year cycle, may effectively depend on the cycle's duration even in that case.

The above result, obtained using oversimplified assumptions, was validated using experimental data. For this purpose, the data of neutron monitor in Climax, one of the longest sets of precise data on GCR flux (1953-2002), was used. This record is shown in Figure 5(A).

The values of cosmic-ray intensity, integrated over each quasi 11-year cycle, are shown in Figure 5(B). Clearly, four cycles of GCR flux is only enough for drawing some preliminary conclusions, especially taking into account that the integral value is appreciably affected by long-term variations of cosmic-ray intensity. However, Figure 5 shows that the difference in integral values of long (1958-1969) and short (1982-1990) peak-type cycles is substantial (more than 30\%). Moreover this difference is at least of the same order as the difference between integrals of peakform cycles (1958-1969, 1982-1990) and adjacent plateau-type cycles (19691982, 1990-2000). This means that if terrestrial climate senses the difference in GCR intensity integrated over peak-form and plateau-form cycles (as indicated by the existence of a bidecadal climatic variation) it should also be sensitive to the length of the quasi-11-year cycle in cosmic-ray flux.

\section{Conclusion}

According to our analysis the appreciable quasi-22-year climatic variation may be linked to the change in galactic cosmic-ray flux, integrated over the full quasi11-year cycle, i.e., to the difference in integrals over peak-type and plateau-type GCR cycles. The more evident presence of bidecadal climatic variability at high latitudes (northern regions) proves its connection with the cosmic-ray intensity. If GCR intensity, integrated over a cycle, indeed affects climate, it must result in a link between the secular variation of climate and the length of the cycle of cosmic-ray flux. This relationship, in turn, should cause a connection between SCL and climate. Our analysis confirms the reality of SCL-climate link for northern Fennoscandia, but does not prove a predominant role of SCL forcing in this region since the century-length variation of NF temperature, which correlates well with smoothed SCL, represents only a minor portion of the total temperature variability. 


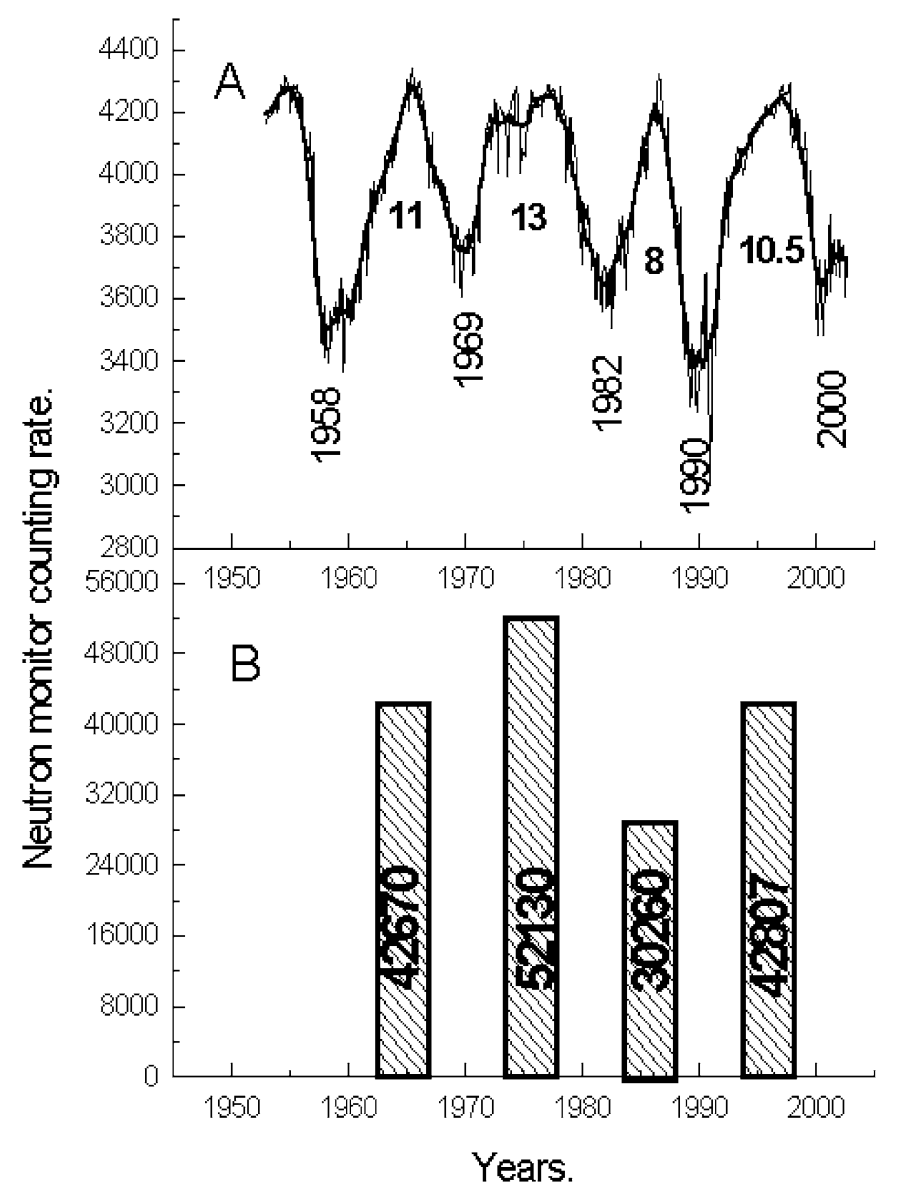

Figure 5. (A) Thin numerals - approximate dates of minima of GCR flux, thick numerals - lengths of cycle in GCR flux. (B) Counting rate of climax neutron monitor integrated over the corresponding cycle in GCR flux.

It is shown that the correlation of century-scale temperature cycles with SCL is stronger if SCL is determined between sunspot maxima. This proves that the NF climate reacts to the duration of cycle of the galactic cosmic ray flux. It is evident that our hypothesis about integral effect of GCR on climate can explain both the bidecadal climatic variability and a connection between secular climatic oscillation and SCL. This hypothesis is in agreement with the assumption of Solanki, Schüssler, and Fligge, (2000), who found a link between SCL and long-term variation of interplanetary magnetic flux (IMF), because IMF effectively modulates cosmic-ray intensity.

The mechanism, which provides sensitivity of climate to the GCR intensity, integrated over a cycle, needs to be clarified. Up to the present, we can only note that optical and electric mechanisms, using GCR as an agent, carrying solar in- 
fluence to earthly atmosphere, are preferable. Undoubtedly, our hypothesis needs further verification. If it is true, the link between climate and the length of cycle of cosmic-ray flux (or SCL) should be stronger at higher latitudes.

\section{Acknowledgements}

This research was done in the frame of an exchange between the Russian and Finnish Academies (project No. 16) and was supported by EU INTAS 2001-0550 grant. In addition it was supported by the program 'Astronomy: nonstationary processes in astronomy' of the Russian Academy and by RFBR grants Nos. 03.02.17505, 03-04-48769. The authors are thankful to an anonymous referee for constructive comments on an earlier version of the paper, which greatly helped to improve it.

\section{References}

Alvarez Pomarez, O. and Perez Doval, J.: 2000, 'The Sun and Space Weather', 24th Meeting of IAU, Manchester, U.K.

Baranyi, T., Ludmany, A., and Coffey, H. E.: 1998, Geophys. Res. Let. 25, 2269.

Beer, J., Soos, F., Lukachyk, Ch., Mende, W., Rodrigues, J., Siegenthaler, U., and Stellmacher, R.: 1994, in: E. Nesme-Ribes (ed.), The Solar Engine and Its Influence on Terrestrial Atmosphere and Climate, NATO ASI Series 25, 221.

Bitvinskas T. T.: 1971, Dendroclimatological Research, Hydrometeoizdat, Moscou (in Russian).

Briffa, K. R., Jones, P. D., Bartholin, T. S., Eckstein, D., Schweingruber, F. H., Karlén, W., Zetterberg, P., and Eronen, M.: 1992, Climate Dynamics 7, 111.

Burroughs, W. J.: 1994, Weather Cycles Real or Imaginary, Cambridge University Press, Cambridge.

Butler, C. J.: 1994, Solar Phys. 152, 35.

Cane, H. V., Wibberenz, G., Richardson, I. G., and von Rosenvige, T. T.: 1999, Geoph. Res. Lett. 26, 565.

Carslaw, K. S., Harrison, R. G., and Kirkby, J.: 2002, Science 298, 1732.

Charakhchian, A. N., Bazilevskaya, G. A., Stozhkov, Yu. I., and Charakhchian T. N.: 1976, Proc. PhIAN 88, 3 (in Russian).

Damon, P. A., and Peristykh, A. N.: 1999, Geophys. Res. Lett. 26, 2469.

Dergachev, V. A., Kocharov, G. E., and Stupneva, A. V.: 1978, Environmental Conditions and Radial Tree Growth, Kaunas, 70-72 (in Russian).

Ermakov, V. I.: 2000, Geomagn. Aeron. 40, 121.

Eronen, M., Zetterberg, P., and Lindholm, M.: 1996, in: J. Roos (ed.), The Finnish Research Programme on Climate Change, Final Report, Publications of the Academy of Finland 4/96, 13.

Friis-Christensen, E., and Lassen, K.: 1991, Science 254, 698.

Haigh, J. G.: 1996, Science 272, 981.

Hathaway, D. H., Wilson, R. M., and Reichman, E. S.: 2002, Solar Phys. 211, 357.

Hibler, W. D., and Johnsen, S. J.: 1979, Nature 280, 481.

Hoyng, P.: 1993, Astron. Astroph. 272, 321.

Jokipii, J. R.: 1991, in C. P. Sonnett, M. S. Giampapa, and M. S. Matthews (eds.), The Sun in Time, University of Arizona Press, Tuscon, pp. 205-220. 
Kocharov, G. E., Konstantinov, A. N., Ostryakov, V. M., and Stupneva, A. V.: 1986, Solar Data 2, 84 (in Russian).

Labitzke, K.: 1987, Geophys. Res. Lett. 10, 877-880.

Larin, I. K.: 2002, Chem. Phys. 21, 45 (in Russian).

Laut, P. and Gundermann, J.: 2000, J. Geophys. Res. 105, 27489.

Lindholm, M, and Eronen, M.: 2000, Geografiska Annaler 82, 527.

Lindholm, M., Eronen, M., Timonen, M., and Meriläinen, J.: 1996, Dendrochronologia 14, 99.

Lindholm, M., Eronen, M., Timonen, M., and Meriläinen, J.: 1999, Ann. Bot. Fennici 36, 119.

Marsh, N. and Svensmark, H.: 2000, Phys. Rev. Let. 85, 5004.

Mitchell, J. M., Stockton, C. V., and Meko, D. M.: 1979, in: B. McNorman, T. Seliga (eds.), SolarTerrestrial Connections, Weather and Climate, D. Reidel Publ. Co., Dordrecht, Holland, pp. 125 -143 .

Mursula, K., Usoskin, I.G., and Kovaltsov G. A.: 2001, Solar Phys. 198, 51.

Ogurtsov, M. G., Kocharov, G. E., Lindholm, M., Eronen, M., and Nagovitsyn, Yu. A.: 2001, Radiocarbon 43, 439.

Ogurtsov, M. G., Kocharov, G. E., Lindholm, M., Merilainen, J., Eronen, M., and Nagovitsyn, Yu.A.: 2002, Solar Phys. 205, 403.

Palle, E., and Butler, C. J.: 2002, J. Atmospheric Solar-Terrest. Phys. 64, 327.

Peristykh, A. N. and Damon, P. E.: 1998, Solar Phys. 177, 343.

Pudovkin, M. I. and Dementeeva, A. L.: 1997, Geomagn. Aeron. 37, 321.

Pudovkin, M. I. and Morozova, A. L.: 1998, J. Atmospheric Solar-Terrest. Phys. 60, 1729.

Pudovkin, M. I. and Morozova, A. L.: 1999, Geomagn. Aeron. 39, 155.

Pudovkin, M. I. and Raspopov, O. M.: 1992, Geomagn. Aeron. 32, 1 (in Russian).

Pudovkin, M. I., and Veretenenko, S. V.: 1996, Adv. Space Res. 17, 161.

Raspopov, O. M., Shumilov, O. I., and Kasatkina, E. A.: 1998, Biophysics 43, 902 (in Russian).

Raspopov, O. I., Shumilov, O. I., Kasatkina, E. A., Jacoby, G., and Dergachev, V. A.: 1997, PhTI, Preprint-1694, St.-Petersburg, 20 p.

Raspopov, O. M., Lovelius, N. V., Shumilov, O. I., and Kasatkina, E. A.: 1998, Biophysics 43, 863 (in Russian).

Russell, C. T. and Mulligan, T.: 1995, Geophys. Res. Lett. 22, 3278.

Rind, D.: 2002, Science 296, 673.

Rivin, Yu.: 1996, Geomagn. Aeron. 36, 531.

Shindell, D., Rind, D., Balachandran, N., Lean, J., and Lonergran, P.: 1999, Science 284, 305.

Solanki, S. K., Schüssler, M., and Fligge, M.: 2000, Nature 408, 445.

Solanki, S. K., Krivova, N. A., Schlüssler, M., and Fligge, M.: 2002, Astron. Astrophys. 396, 1029.

Stewart, J. Q., and Panofsky, A. A.: 1932, Astrophys. J. 88, 385.

Stozhkov, Yu. I., Ermakov, V. I., and Pokrevski, P. E.: 2001, Izv. RAN (Ser. Phys.) 65, 406 (in Russian). Svensmark, H., and Friis-Christiensen, E.: 1997, J. Atmospheric Solar-Terrest. Phys. 59, 1225.

Tinsley, B. A.: 2000, Space Sci. Rev. 94, 231.

Tinsley, B. A. and Deen, G. W. 1991, J. Geophys. Res. 96, 22283.

Veretenenko, S. I. and Pudovkin, M. I.: 1995, Geomagn. Aeron. 34, 463.

Veretenenko, S. I., and Pudovkin, M. I.: 1997, J. Atmospheric Solar-Terrest. Phys. 59, 1739.

Veretenenko, S. V., and Pudovkin M. I.: 2000, J. Atmospheric Solar-Terrest. Phys. 62, 567.

Vitinski, Yu. I., Ohl, A. I., and Sazonov, B. I.: 1976, in E. R. Mustel (ed.), The Sun and Earth's Atmosphere, Nauka, Leningrad, 352 pp. (in Russian).

Ulich, T.: 1996, M. of Sc. Thes., University of Oulu.

Zhou, K. and Butler, C. J.: 1998, J. Atmospheric Solar-Terrest. Phys. 60, 1711. 